Orthopäde 2021 · 50:605-607

https://doi.org/10.1007/s00132-021-04129-1

Angenommen: 8. Juni 2021

(c) Springer Medizin Verlag $\mathrm{GmbH}$, ein Teil von Springer Nature 2021

\section{Neuromuskuläre Skoliosen}

\author{
Christoph-E. Heyde ${ }^{1}$ M. Putzier ${ }^{2}$ \\ ${ }^{1}$ Klinik u. Poliklinik für Orthopädie, Unfallchirurgie u. Plastische Chirurgie, Bereich Wirbelsäulenchirurgie, \\ Universitätsklinikum Leipzig AöR, Leipzig, Deutschland \\ ${ }^{2}$ Centrum für Muskuloskeletale Chirurgie, Bereich Wirbelsäulenchirurgie, Charité - Universitätsmedizin \\ Berlin, Berlin, Deutschland
}

Neuromuskuläre Deformitäten der Wirbelsäule entstehen auf dem Boden verschiedenster angeborener oder erworbener Erkrankungen des zentralen Nervensystems, des ersten und zweiten Motoneurons und/ oder der Skelettmuskulatur. Viele dieser Erkrankungen oder Erkrankungsgruppen führen mit mehr oder minder großer Wahrscheinlichkeit bereits im Wachstumsalter zu überwiegend skoliotischen Deformitäten, seltener aber auch zu Kyphosen oder kombinierten Fehlstellungen.

Diese neuromuskulär bedingten Skoliosen sind deutlich seltener als idiopathische, zeigen aber eine sehr eigene und krankheitsspezifische Dynamik sowie Progredienz, was spezielle Kenntnisse für die richtige Beurteilung und notwendige Therapieentscheidungen erforderlich macht.

Neben den Auswirkungen auf den gesamten Stütz- und Bewegungsapparat sind die zugrundeliegenden Erkrankungen häufig sowohl mit weiteren physischen als auch mit geistigen Beeinträchtigungen bis hin zu ausgeprägten Behinderungen verbunden. Somit ist ein ganzheitlicher Behandlungsansatz in dafür ausgewiesenen Zentren notwendig.

Unter den vielen dabei zu berücksichtigen Teilaspekten muss eine sich entwickelnde Wirbelsäulendeformität früh erfasst, konsequent kontrolliert und schließlich therapiert werden. Die verschiedenen Krankheitsgruppen variieren dabei stark in ihrer Wahrscheinlichkeit, Deformitäten auszubilden, und auch die zu erwartende Progredienz ist ursachenbedingt sehr unterschiedlich ausgeprägt.

Das vorliegende Themenheft widmet sich diesen Deformitäten der Wirbelsäule, ohne bei der Fülle der ursächlichen Erkrankungen und all ihrer Spezifika auch nur im Ansatz Anspruch auf Vollständigkeit erhe- ben zu können. Dennoch ist es Ziel dieses Heftes, einen möglichst weiten Blick auf die Besonderheiten neuromuskulärer Skoliosen zu werfen. Dafür konnten auf diesem Gebiet erfahrene Autoren gewonnen werden, denen wir für ihr Engagement an dieser Stelle ausdrücklich danken möchten.

Im ersten Artikel wird auf ätiologische, epidemiologische und biomechanische Grundlagen neuromuskulär bedingter Wirbelsäulendeformitäten eingegangen. Die beiden nachfolgenden Artikel widmen sich den konservativen Therapiemöglichkeiten, einschließlich der erforderlichen orthopädietechnischen Versorgungsmöglichkeiten. Diese ersten Kapitel unterstreichen die Notwendigkeit eines ganzheitlichen und interdisziplinären Behandlungsansatzes als Grundlage einer lebensbegleitenden Therapie.

Im darauffolgenden Artikel wird zur Rolle der operativen Versorgung Stellung genommen, insbesondere unter dem Aspekt der Indikation und des günstigsten Zeitpunktes. Hier wird aufgezeigt, dass ein zeitgerechtes Eingreifen, beruhend auf einer fundierten Kenntnis der Prognose, segensreich sein kann.

Nachfolgend wird detailliert auf spezielle Krankheitsbilder und das jeweilig erforderliche therapeutische Vorgehen eingegangen. Dabei werden die Muskeldystrophie Typ Duchenne, zentral bedingte Paresen sowie spinale Muskelatrophien betrachtet. Die schwer zuzuordnenden Deformitäten auf der Grundlage einer Neurofibromatose Typ 1 werden ebenfalls besprochen. Diese Krankheitsbilder und -gruppen stellen zwar nur einen Ausschnitt aus dem Bereich sekundärer Wirbelsäulendeformitäten dar, sind aber bewusst ausgewählt und ba- 
sierend auf dem aktuellen Wissensstand vorgestellt worden, da sie alle Besonderheiten hinsichtlich der konservativen, aber insbesondere auch der operativen Versorgungsnotwendigkeiten zeigen.

Das vorliegende Themenheft arbeitet somit Charakteristika neuromuskulärer Skoliosen heraus, wobei der Bogen bewusst von den allgemeinen Grundlagen über die konservative und die orthopädietechnische Versorgung bis hin zur operativen Therapie gespannt wurde. Trotzdem repräsentiert die Wirbelsäule nur einen - wenn auch wichtigen - Teilaspekt dieser ja überwiegend systemischen Erkrankungen. Somit muss der ganzheitliche und lebensbegleitende Aspekt der therapeutischen Herangehensweise bei allen erforderlichen Maßnahmen immer aufs Neue berücksichtigt werden.

\section{Korrespondenzadresse}

Prof. Dr. Christoph-E. Heyde

Klinik u. Poliklinik für Orthopädie,

Unfallchirurgie u. Plastische Chirurgie, Bereich Wirbelsäulenchirurgie, Universitätsklinikum Leipzig AöR

Liebigstraße 20, 04103 Leipzig, Deutschland christoph-eckhard.heyde@uniklinik-leipzig.de

Interessenkonflikt. C.-E. Heyde erhält Royalties von Medacta Int. M. Putzier gibt an, dass kein Interessenkonflikt besteht.

Holm-Torsten Klemm, Michael Karl-Heinz Wich

\section{Ärztliche Begutachtung - Strukturierte Curriculare Fortbildung nach den Vorgaben der Bundesärztekammer, Module I und II \\ Strukturierte Curriculare Fortbildung nach den Vorgaben der Bundesärztekammer, Module I und II}

De Gruyter 2021, 397 S., 21 Abb., 13 Tab., (ISBN: 9783110693355$),$ 99,95 EUR

Es den Herausgebern gelungen, ein umfassendes Werk zur ärztlichen Begutachtung zu erstellen, welches sich streng an den Vorgaben der Bundesärztekammer zur strukturierten curricularen Fortbildung (SCF) „medizinische Begutachtung" orientiert. Dieses Curriculum wurde 2003 eingeführt, 2008 und 2014 überarbeitet.

Die strukturierte curriculare Fortbildung wendet sich an alle Ärzte, die Interesse haben, neben ihren medizinisch-fachlichen Qualifikationen ihre Kenntnisse in der Gutachtenerstellung zu erhalten und zu vertiefen. Das Curriculum umfasst 64 Stunden und besteht aus 3 Modulen. Neben dem engen Bezug zu den Modulen I und II des oben genannten Curriculums erläutern erstmals Mediziner gemeinsam mit Juristen die Anforderungen, welche an ein ärztliches Gutachten gestellt werden. In diesem Buch findet man erstund einmalig eine Synthese juristischen und ärztlichen Denkens. Dieses Werk spiegelt die jahrzehntelange Erfahrung der Autoren wider und präsentiert einen hervorragenden Leitfaden für den Arzt als Gutachter. Es findet sich eine sehr verständliche und klare Gliederung, beginnend mit den Grundlagen ärztlicher Begutachtung über praktische Hinweise und rechtliche Grundlagen der ärztlichen Begutachtung. Naturgemäß wird Bezug genommen auf die Begutachtung der Leistungsfähigkeit, der Rehabilitation und des Schwerbehindertenrechtes. Im Modul I b werden die Kausalitäts- und Beweisregeln im Straf-, Zivil- und Sozialrecht wie auch die Themen Haftpflichtversicherung, gesetzliche und private Unfallversicherung, soziales Entschädigungsrecht und Begutachtung im Bereich der Arzthaftung umfassend diskutiert. In gleicher Weise werden dann im Modul I c die Pflege- und private Krankenversicherung, sowie Berufsunfähigkeits- (Zusatz) Versicherungen, spezielle Begutachtungsfragen aus der Praxis und unter speziellen Blickwinkeln immer wieder mit den Hinweisen auf die Widersprüchlichkeit zwischen medizinischen Erkenntnissen und rechtlichen Vorgaben abgehandelt. Das Modul II beschäftigt sich dann mit dem komplizierten Thema der Beschwerdevalidierung aus verschiedenen fachspezifischen Blickwinkeln der Orthopädie, Unfallchirurgie, Neurologie und Psychiatrie. Auch finden die allgemeine Schmerzbegutachtung sowie die immer aktueller werdenden kultursensiblen Aspekte von Begutachtung und Forensik Beachtung. Zuletzt werden auch Fallstricke der ärztlichen Begutachtung und praktische Hinweise zu Einzelaspekten umfassend dargestellt.

Insgesamt findet sich im Werk ein allumfassender gutachtlicher Überblick mit der tatsächlich einmaligen Vereinigung aus juristischen und ärztlichen Denkens. Perfekt gelungen ist damit ein Begleitbuch für Kurs SCF nach den Vorlagen der Bundesärztekammer mit einer außergewöhnlichen Praxisnähe und immer wieder anschaulichen Beispielen zu jedem Thema und dazu passender TakeHome-Message für jeden Themenschwerpunkt.

Dieses Buch der ärztlichen Begutachtung ist ein MUSS für jedem Arzt in Weiterbildung wie auch den Facharzt, der sich mit der medizinischen Begutachtung beschäftigt und es ist ein guter Wegbegleiter für die curriculare Fortbildung der BÄK.

Dr. med. Wolfgang Willauschus, Bamberg 
Hier steht eine Anzeige.

黑 Springer 